\title{
Impact of fume particles in the keyhole vapour
}

\author{
J.Volpp ${ }^{1}$ (1)
}

Received: 16 August 2018 / Accepted: 18 December 2018 / Published online: 5 January 2019

(c) The Author(s) 2019

\begin{abstract}
During laser material processing, the energy of the laser beam needs to be efficiently and constantly transported to the processing zone to guarantee constant processing. However, spatters or ejected particles from the keyhole can absorb and scatter the laser energy leading to inhomogeneous heat input and can initiate defect occurrence like pore formation. The impact of ejected particles from the keyhole on the energy transport of the laser beam is not completely understood since they are difficult to observe due to the small size and high speeds of the ejections. In this work, the particle characteristics were derived from a simulation of the keyhole wall movement. The behavior of the calculated particles in a side shielding gas jet was calculated to derive the height, at which the particles leave the laser beam and are not interrupting the laser energy transfer to the processing zone. Low impulse values of the particles were calculated e.g., at defocusing positions slightly underneath the material surface, where also highest melt pool sizes were found. These observations indicate that the fume particles can be one reason to limit the energy delivery. An efficiency increase can be achieved by adjusting the keyhole parameters to a more stable keyhole.
\end{abstract}

\section{Introduction}

Laser deep penetration welding is a highly complex process due to the occurrence of high temperatures leading to melting, vaporization and the interaction of solid, liquid and vaporized material. The characteristic vapor channel, the keyhole, existing during processing, is kept open during processing due to the recoil pressure induced by the vaporization of the keyhole walls due to laser irradiation. The vapor outflow is known to be unstable, e.g., pore formation requires a reversed gas flow of ambient gas into the keyhole (e.g., [1]). Fabbro et al. [2] found that depending on the process parameters the vapor outflow can show a pulsed characteristic. The keyhole itself is highly dynamic showing moving melt waves on the keyhole front wall [3] and small humps appearing and moving at frequencies up to $1 \mathrm{MHz}$ [4]. Those fluctuations are thought to be the origin of typical defects, such as spatters and pores (e.g., demonstrated by Volpp [5]). Besides spatter emission, the vapor plume appears above the keyhole exit due to vaporization happening on the keyhole walls during processing. This dynamic

\section{J. Volpp \\ jorg.volpp@1tu.se}

1 Department of Engineering Sciences and Mathematics, Luleå University of Technology, 97187 Luleå, Sweden vapor characteristics could be related to fluctuations in the keyhole [6], which means that the keyhole behavior seems to define the vapor outflow characteristics.

When welding with a $\mathrm{CO}_{2}$-laser (wavelength $10.6 \mu \mathrm{m}$ ) instead of solid-state lasers (wavelength around $1 \mu \mathrm{m}$ ), gases in the laser beam can be even ionized leading to plasma creation. The plasma can absorb laser beam energy, which cannot be transported to the processing zone and can interrupt or even stop the processing [7]. It has been shown that when processing with solid-state lasers, plasma effects can be neglected [8]. Kawahito et al. [9] used spectroscopic measurements when welding stainless steel with a $10-\mathrm{kW}$ fiber laser and found that the induced plume mainly consists of the alloying elements from the base material and that the plume is not highly ionized to form a plasma.

However, it was observed that the plume affects the laser energy transport to the keyhole. Michalowski et al. [10] observed that more than $30 \%$ of the energy of the laser beam can be lost due to scattering and absorption in the plume. Scholz et al. [11] observed that fluctuations of the particle appearance lead to inherent variations of the energy delivery to the processing zone. This, in consequence, could in turn influence the keyhole wall fluctuations.

Shcheglov et al. [12] observed two characteristic parts of the plume when laser welding mild steel (Fig. 1). The lower plume glows bright and shows fluctuation frequencies of 


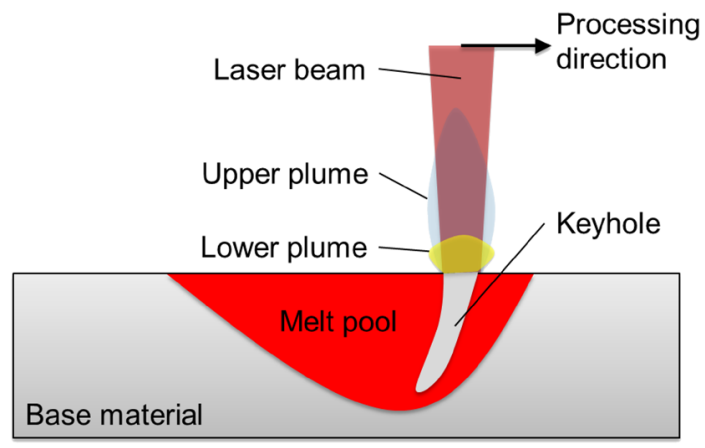

Fig. 1 Process appearance according to Shcheglov et al. [12]

several $\mathrm{kHz}$, while the upper part shows weaker emissions and is related to the laser beam path.

The size of the particles, which are present in the plume, were measured by different authors (Fig. 2).

The detected particle sizes vary from $10 \mathrm{~nm}$ (Scholz et al. [13]) to $192 \mu \mathrm{m}$ (Michalowski et al. [10]). Due to the wide range, the particles could origin from different mechanisms. The small particles can be related to the vaporization of material from the keyhole wall and the re-condensation during their outflow on other particles (e.g., [13]). The bigger particles could be also micro-spatters detached from the keyhole walls. Particles in the laser beam, in general, influence the laser energy delivery by absorption of energy on the particle surface or scattering (e.g., [14]), which both leads to a lower energy input into the keyhole. The detection of the particles was partly possible; their origin could not be sufficiently observed yet. Modeling approaches were conducted to simulate the behavior of the keyhole and melt pool. Pointto-point calculations enabled to model keyhole geometries depending on processing parameters (e.g., [15]) or could help predict the porosity [16]. Simplified models can help to predict the keyhole depth [17], which might not fully represent the dynamic observed behavior of the keyhole (e.g., observed in ice [18] or during copper welding [19]).

It is known that a shielding gas flow can blow away the particles, which are ejected from the keyhole, which can lead to a more efficient and constant energy absorption. Especially when processing with a $\mathrm{CO}_{2}$ laser, where plasma forming is expected, an application of shielding gas can significantly

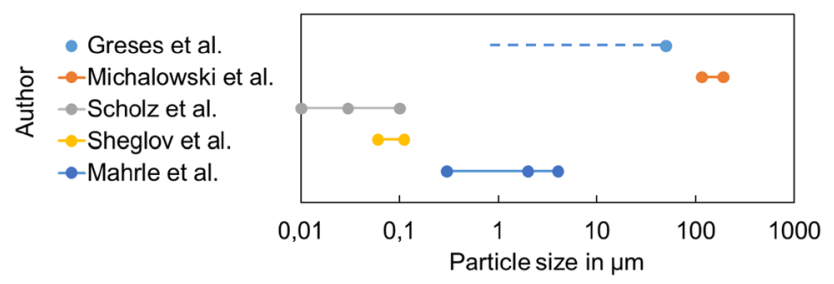

Fig. 2 Measured particle sizes in the keyhole vapor plume improve the energy input by blowing the plasma away from the laser beam circumference. When processing with a fiber laser, Mahrle et al. [20] found that a gas flow of $2 \mathrm{~m} / \mathrm{s}$ applied by a shielding gas nozzle from a rectangular position to the straight upwards directed vapor plume is sufficient to suppress interactions between the laser beam and the plume. It seems that a reduction of the particle density in the plume reduces the energy absorption and/or scattering by the plume.

However, it is not known, where and how these particles are created and how they influence the welding results. It is known that at negative focal positions (focal point below the material surface) can be beneficial to achieve a higher heat input into the keyhole, which is assumed to be related to increased multiple reflections in the keyhole or a more stable keyhole behavior (e.g., [21]). However, it is possible that the characteristics of the vapor can affect the energy delivery as well. Knowledge about the creation and transporting of those particles could help find methods to avoid particle creation in general and thereby increase the process efficiency. In addition, knowledge about the occurrence of particle sizes and their impact on the laser beam absorption could help define critical particle sizes and open the discussion of influencing the keyhole wall characteristics to avoid creating critical particle sizes that induce process changes and defects. Therefore, in this work, the possible origin, their size distribution and characteristics of particles that are created on the dynamic keyhole wall are examined and related to experimental observations.

\section{Methodology}

\subsection{Experiment conduction}

A single-mode fiber laser (IPG YLR-1000SM) was used to produce the laser beam, which was collimated $(160 \mathrm{~mm}$ focal length) and focused (560 $\mathrm{mm}$ focal length). The resulting focal beam diameter was $52 \mu \mathrm{m}$ at a Rayleigh length of $1.4 \mathrm{~mm}$. The focal spatial laser intensity distribution shows a Gaussian beam profile. Reference parameters were defined (Table 1).

Based on these parameters, variations of the laser power (from 800 to $1000 \mathrm{~W}$ ), the welding speed (from 0.5 to $2 \mathrm{~m} / \mathrm{min}$ ) and the focal position ( -1 to $+1 \mathrm{~mm}$ ) were conducted. No shielding gas was used. Base material was stainless steel 1.4301, while specimens were of the size $40 \mathrm{~mm} \times 6 \mathrm{~mm} \times 100 \mathrm{~mm}$. $80 \mathrm{~mm}$ long weld seams were produced.

Table 1 Reference parameters

\begin{tabular}{llll}
\hline Laser power & $P_{\mathrm{L}}$ & 1 & $\mathrm{~kW}$ \\
\hline Welding velocity & $\mathrm{v}$ & 1 & $\mathrm{~m} / \mathrm{min}$ \\
Focal position & $z_{\mathrm{f}}$ & -2 & $\mathrm{~mm}$ \\
\hline
\end{tabular}




\subsection{Weld seam evaluation}

Cross-section polishes were produced to evaluate the weld seam dimension. The weld seam area was derived as visualized in Fig. 3.

\subsection{Keyhole modeling}

A simplified semi-analytical model was developed to calculate the keyhole properties, which consist of two parts. An analytical model calculates the quasi-static conditions of the keyhole, while a subsequent time-dependent calculation simulates the dynamic behavior of the keyhole wall movement. The model was described in detail in [22].

The quasi-static model is based on a segmentation of the keyhole into ten cylindrical elements (Fig. 4). Initially, the depth of the keyhole must be given and was derived from experimental measurements. Based on the laser beam caustic, the energy input in every element is calculated assuming Fresnel absorption on the inclined keyhole walls (Fig. 4).

The spatial laser intensity distribution of the laser beam was considered a Gaussian distribution $i_{g s}$ and was calculated in every element depending on the actual beam diameter and was calculated using the following equation:

$i_{\mathrm{gs}}(r)=\frac{P_{\mathrm{L}}}{\pi \cdot r_{\mathrm{L} 0}^{2}} \cdot e^{-\left(\frac{2 \cdot r}{r_{L 0}}\right)}$,

with the laser power $P_{\mathrm{L}}$, the beam radius $r$ and the focal laser beam radius $r_{\mathrm{L} 0}$.

The energy balance equation was considered including the contribution of the energy input from the laser beam and energy losses from heat conduction into the surrounding



Fig. 3 Measuring the weld seam area in cross-section polishes

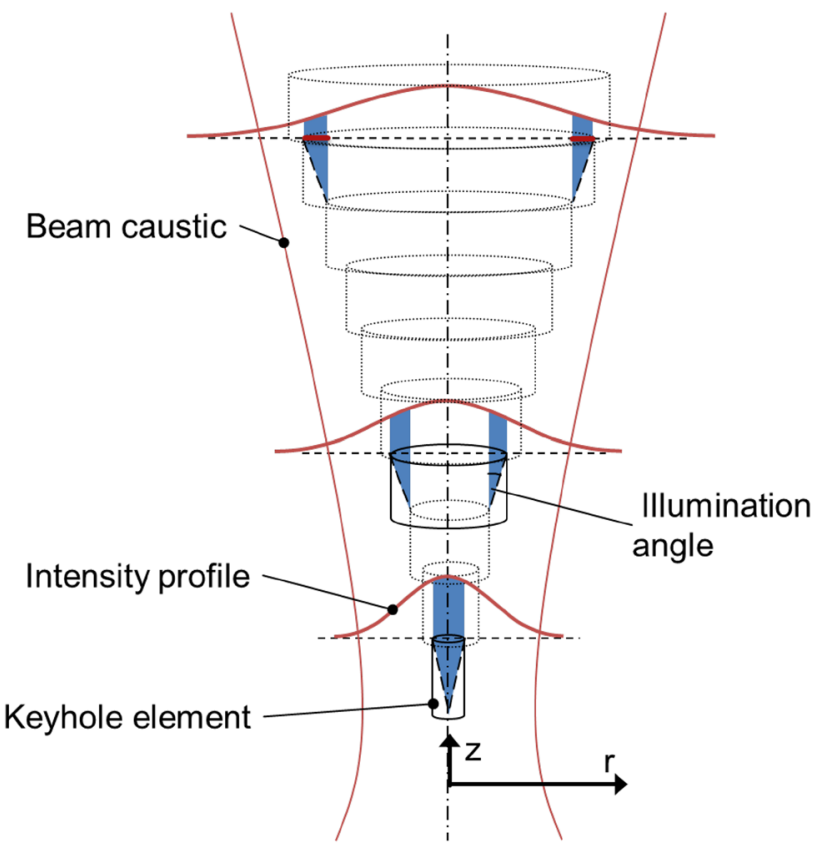

Fig. 4 Keyhole sectioning for heat input modeling

material and due to vaporization effects. The solution of the energy equation gives the actual temperature in each keyhole element. The temperature values are needed to calculate the pressure balance equation. In all keyhole elements, the vapor pressure was calculated based on the actual temperature in the element. The vapor pressure keeps the keyhole element open against pressure of the surrounding melt pool due to surface tension. The pressure balance calculation gives the actual radius of the cylindrical keyhole element. Therefore, the quasi-static keyhole shape was derived, which was used as boundary condition for the temporal calculation.

The dynamic model used the quasi-static geometrical keyhole data as starting conditions of the movement of the keyhole walls of the single elements. To describe the keyhole elements as a dynamic system, a set of differential equations was used. The pressure differential equation contains the impact of evaporation and the change of the element volume at element expansion or shrinkage. Evaporation was based on the actual energy input by the laser beam, considering Fresnel absorption effects and multiple reflections inside the keyhole using an iterative routine. The second differential equation described the radius development of the elements, including the effects of the ablation pressure, which increases the radius and the counteracting surface tension pressure initiated by the melt pool and the bulging of the melt pool at the surface. This description gives the characteristics (frequencies, amplitudes) of each single keyhole element. The solution of the equations (solver: ode45), calculated in Matlab Simulink (Version R2009a), gives the temporal development of the dynamic properties of 
the keyhole during processing. Table 2 shows the parameters used for the calculation.

The calculated keyhole wall movements were compared to acoustic emissions recorded by a microphone during the processing (Fig. 5).

The average of the dynamic characteristic properties was evaluated at different parameter sets to be compared to the experimental evaluations. The dynamic values were used to calculate the impulse I of the particle as

$l=\frac{4}{3} \cdot \pi \cdot\left(\frac{A}{2}\right)^{2} \cdot \rho_{\mathrm{met}} \cdot v_{\mathrm{part}}$,

with the amplitude A, the density $\rho_{\text {met }}$ and the particle speed $v_{\text {part }}$. The particle speed was calculated assuming the maximum wall speed being transferred onto the particle by

$\nu_{\text {part }}=2 \cdot \pi \cdot f \cdot A$.

\subsection{Particle flow modeling}

An additional model was developed to analyze the metal particle flow paths that were ejected from the keyhole walls after exiting the keyhole at a defined side gas application. The particle paths were calculated at the following assumptions:

- all particles exit the keyhole vertically to the material surface, which can be assumed due to the strong vapor outflow from the keyhole,

- all particles are perfect spheres due to the surface tension.

When exiting the keyhole, the ejected particle experiences the flow of a horizontally applied gas jet with a gas flow speed of $2 \mathrm{~m} / \mathrm{min}$ (Fig. 6).

This side gas flow induces a pressure $p$ on the particle of

$p=\frac{\rho_{\text {air }}}{2} \cdot\left(v_{\text {air }}\right)^{2}$,

with $\rho_{\text {air }}$ being the density of the gas and $v_{\text {air }}$ being the speed of the air. The pressure applied on the projected area of a particle $B$

$B=\pi \cdot\left(\frac{A}{2}\right)^{2}$,

Table 2 Selected material properties (mainly from [23])

\begin{tabular}{llll}
\hline Parameter & Symbol & Value & Unit \\
\hline Density & $\rho_{\text {met }}$ & 7.87 & $\mathrm{~g} / \mathrm{cm}^{3}$ \\
Thermal conductivity & $k$ & 80.4 & $\mathrm{~W} /(\mathrm{m} * \mathrm{~K})$ \\
Specific heat capacity & $c_{\mathrm{p}}$ & 449 & $\mathrm{~J} /(\mathrm{kg} * \mathrm{~K})$ \\
Boiling temperature & $T_{\mathrm{b}}$ & 2861 & ${ }^{\circ} \mathrm{C}$ \\
Melting temperature & $T_{\mathrm{m}}$ & 1538 & ${ }^{\circ} \mathrm{C}$ \\
Latent heat (vaporization) & $L_{\mathrm{v}}$ & 6260 & $\mathrm{~kJ} / \mathrm{kg}$ \\
Latent heat (melting) & $L_{\mathrm{m}}$ & 268 & $\mathrm{~kJ} / \mathrm{kg}$ \\
Surface tension coefficient (at $\left.T_{\mathrm{s}}\right)[24]$ & $\gamma$ & 1909 & $\mathrm{mN} / \mathrm{m}$ \\
\hline
\end{tabular}

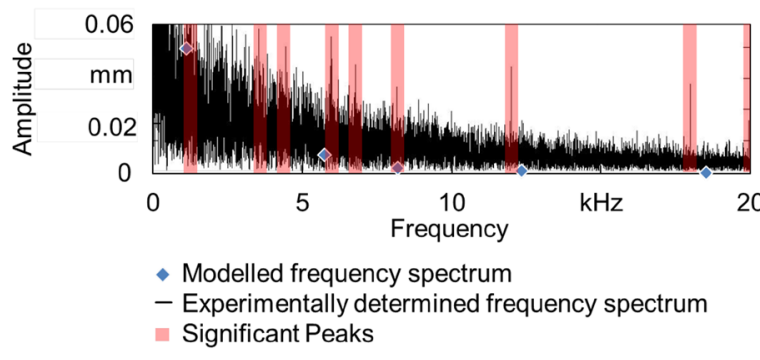

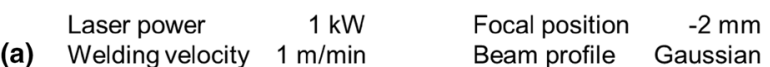

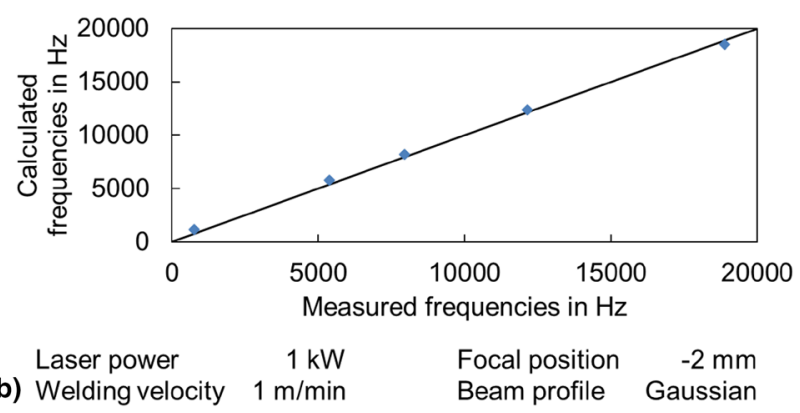

Fig. 5 Comparison of acoustic measurements and calculated frequency peaks from the analytical model, a exemplary frequency spectrum and $\mathbf{b}$ comparison of identified correlated frequencies

induces a force $F$

$F=p \cdot B$,

on the particle with the massm

$m=\rho_{\text {met }} \cdot \frac{4}{3} \cdot \pi \cdot\left(\frac{A}{2}\right)^{3}$,

leading to an acceleration $a$ of the particle

$a=\frac{F}{m}$.

According to the relations at accelerated movement, the time till leaving the laser beam

$t=\sqrt{\frac{2 \cdot\left(2 \cdot r_{\mathrm{L} 0}\right)}{a}}$,

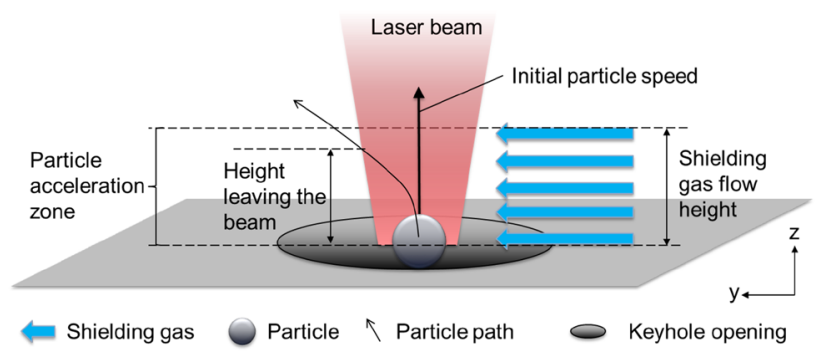

Fig. 6 Sketch of a particle outflow from the keyhole opening and definition of model parameters 
when assuming a laser beam with a constant diameter $\left(2 \cdot r_{\mathrm{L} 0}\right)$, which can be assumed since the calculation is done close to the focal plane.

When considering the initial speed of the particle assumed in vertical upward direction when exiting the keyhole $v_{\text {part }}$, the height $h$ above the material surface was determined, at which the particle leaves the laser beam and is not an obstacle for the transfer of laser energy to the processing zone anymore to

$h=v_{\text {part }} \cdot t$.

\section{Results}

\subsection{Weld seam characteristics}

The weld seam areas at different parameter sets were evaluated as described in the Methods for comparison of different processing parameters. Figure 7 shows the measured weld seam areas derived from cross-section polishes.

At a higher heat input (lower velocity or higher laser power), the weld seam area increases. At varied focal position, the highest value was found at $-1 \mathrm{~mm}$ defocusing.

\subsection{Keyhole characteristics}

The keyhole simulation calculated the characteristics of the temporal keyhole wall development in the single keyhole elements. The average of all amplitudes and frequencies calculated in all keyhole elements including the range of occurring data points are visualized in Figs. 8 and 9.

The occurring amplitudes (Fig. 8) show the lowest values at defocusing underneath the material surface, while the frequencies of the keyhole wall movement are comparably

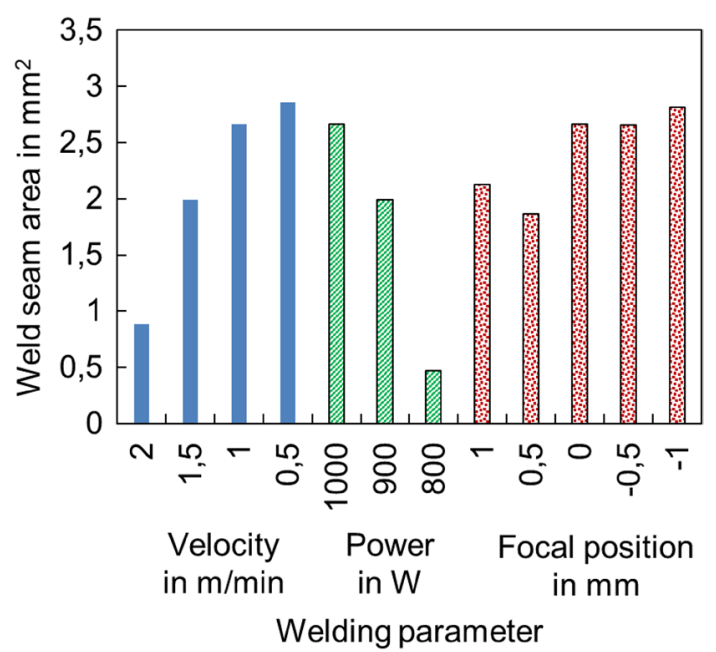

Fig. 7 Weld seam areas determined from cross-section polishes at different parameter sets based on the reference parameters high (Fig. 9). These results describe the dynamic movement of the keyhole wall elements depending on the processing parameters.

From the calculated frequencies and amplitudes of the keyhole elements, the impulse was calculated using Eq. 2 (Fig. 10). The impulse results from the keyhole wall movement and shows lowest values at high velocities and defocusing underneath the material surface.

\subsection{Particle paths}

The calculation of the particle path of the material exiting the keyhole with the vapor when applying a side gas jet was conducted. This simple approach makes it possible to determine the height above the material surface, at which a particle with the diameter of the keyhole wall amplitude in the keyhole at its calculated impulse (Fig. 10) leaves the laser beam (Fig. 11) and can give indications how much material is inside the vapor plume that can affect the laser energy transmission.

At lower welding speeds, particles can be removed from the laser beam by shielding gas more efficiently, while the

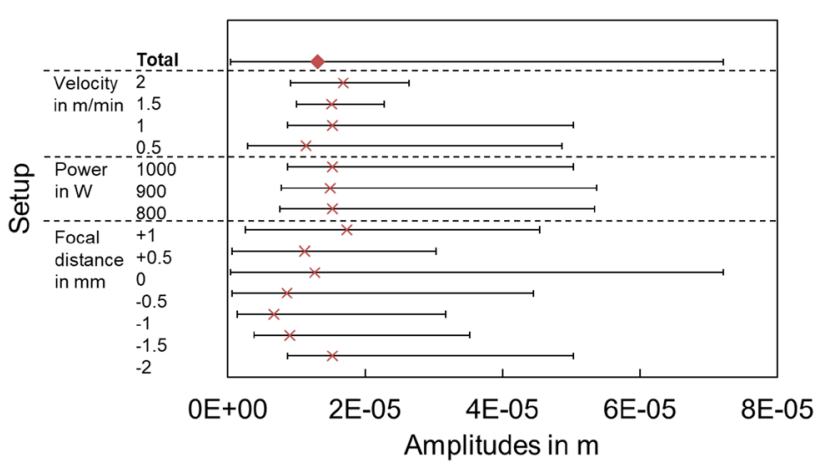

Fig. 8 Calculated keyhole wall amplitudes in all keyhole elements at different processing parameters

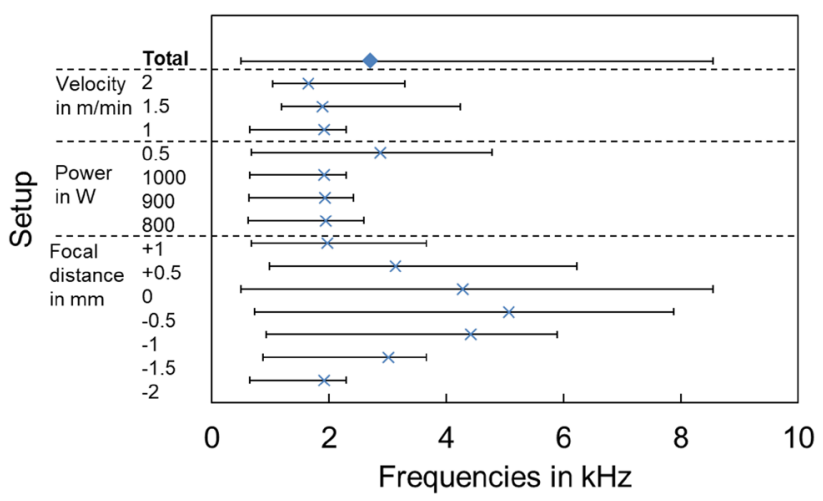

Fig. 9 Calculated keyhole wall frequencies in all keyhole elements at different processing parameters 


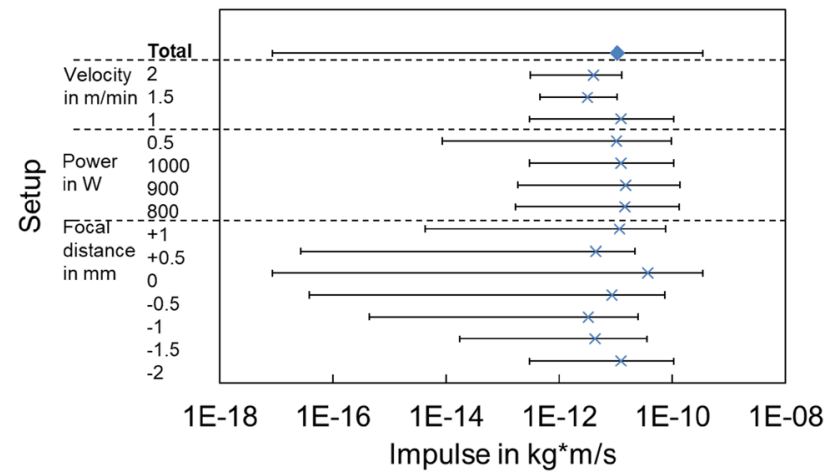

Fig. 10 Calculated impulse of the ejected particles from keyhole wall fluctuations in all keyhole elements and observed parameter sets

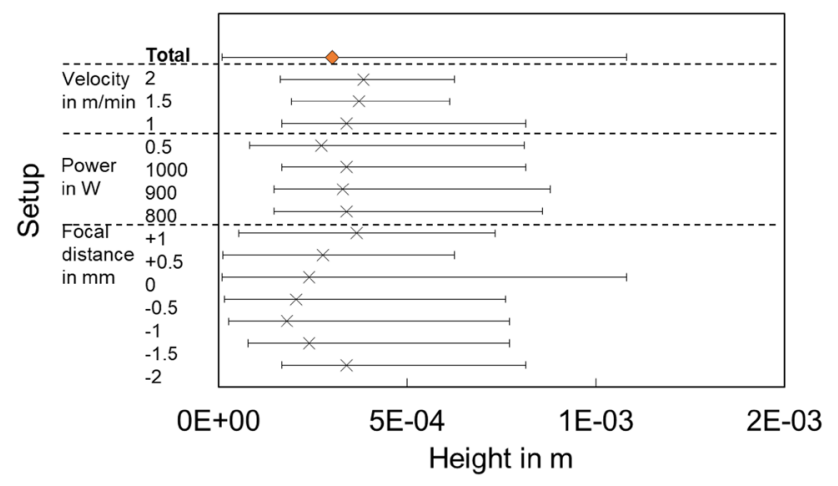

Fig. 11 Calculated height above the material surface when ejected particles from keyhole wall movements leave the laser beam due to a side shielding gas flow

laser power showed minor impact on the particle paths. At a focal position slightly below the material surface (negative values), the particle height was calculated in average the lowest, which indicates the setting with the most efficient removal of particles from the laser beam.

\section{Discussion}

In the literature, it was often observed that when positioning the laser beam focal position slightly underneath the material surface, a higher energy input efficiency into the keyhole can be achieved (e.g., [21]). In the actual work, this phenomenon could be also seen in the measured weld seam areas (Fig. 7). Possible reasons for this phenomenon could be:

- an increased energy absorption due to increased amounts of multiple reflection,

- a more stable keyhole behavior, leading to a more constant absorption on the keyhole walls, or,

- the effect of different ejected plume particles and their impact on energy absorption and scattering.
Especially, the plume content and its impact on the processes are barely considered, since the measuring of the particle characteristics can be difficult due to high temperatures and pressures and the fast processes, as well as the limited access to their origin.

The proposed model in this paper calculated the expected ranges of particles that can be ejected from the keyhole wall at its dynamic movement. The keyhole wall movement creates the material bulging into the keyhole area, while the created vapor gas flow can be able to detach the small particles from the keyhole wall [5].

Since in other works (Fig. 2) a big range of particle sizes were found in the plume, it can be assumed that different origins are possible. Small particles are most likely re-condensated from vapor content, the slightly larger particles could also origin from keyhole wall detachments as micro-spatters as derived from the results also in this work.

The simulation results in this work show that different characteristics (especially the impulse) of detached particles can occur depending on the process parameters. Since usually shielding gas is used to blow away the plume, a standard shielding gas flow was considered that blows away the plume including the particles that are ejected from the keyhole. In general, a higher impulse leads to a later leaving of the laser beam and can therefore lead to more energy absorption or scattering in the plume and therefore to a lower laser energy transport to the keyhole walls.

Tendencies show that at a focal position underneath the material surface (Fig. 10), the particles show a comparably low impulse, which leads to a fast removal of the particles by the shielding gas. This observation shows that the particle characteristics can possibly be an impacting factor on the energy absorption efficiency and can partly explain the increased absorption at negative focal positions. This observation shows that the particle scattering can have an impact on the laser energy absorption leading to different keyhole characteristics and absorption mechanisms.

\section{Conclusions}

Comparing experimental results of weld seam area dimensions, which are a measure of energy absorption, and particle detachment simulations from the keyhole wall, it can be concluded that the particles that are ejected from the keyhole wall can affect the laser beam energy transport to the processing zone. It seems that the ejected particles are part of the mechanism that leads to the increased energy absorption when e.g., the focal position is positioned below the material surface. 
Acknowledgements The author is grateful for the financial support by the Senatorin für Wissenschaft, Gesundheit und Verbraucherschutz of the country of Bremen.

Open Access This article is distributed under the terms of the Creative Commons Attribution 4.0 International License (http://creativeco mmons.org/licenses/by/4.0/), which permits unrestricted use, distribution, and reproduction in any medium, provided you give appropriate credit to the original author(s) and the source, provide a link to the Creative Commons license, and indicate if changes were made.

\section{References}

1. S. Pang, X. Chen, X. Shao, S. Gong, J. Xiao, Dynamics of vapor plume in transient keyhole during laser welding of stainless steel: local evaporation, plume swing and gas entrapment into porosity. Opt. Lasers Eng. 82, 28-40 (2016)

2. R. Fabbro, S. Slimani, I. Doudet, F. Coste, F. Briand, Experimental study of the dynamical coupling between the induced vapour plume and the melt pool for Nd-Yag CW laser welding. J. Phys. D Appl. Phys. 39, 394-400 (2006)

3. P. Berger, R. Schuster, H. Huegel, T. Graf, Moving humps at the capillary front in laser welding. in Proc. of the 29th International Congress on Applications of Lasers and Electro-Optics (ICALEO), paper 106 (2010)

4. A.F.H. Kaplan, S.M. Ramiz, Absorption peaks depending on topology on the keyhole front and wavelength. J. Laser Appl. 27(1 - S29012-9), S29012 (2015)

5. J. Volpp, Formation mechanisms of pores and spatters during laser deep penetration welding. J. Laser Appl. 30(1), 012002 (2018)

6. J. Volpp, D. Freimann, Indirect measurement of keyhole pressure oscillations during laser deep penetration welding. in Proc. of the 32nd International Congress on Applications of Lasers and Electro-Optics (ICALEO), paper 1301, 334-340 (2013)

7. S. Katayama, Y. Kawahito, M. Mizutani, Latest progress in performance and understanding of laser welding. Phys. Proc. 39, 8-16 (2012)

8. I. Miyamoto, Laser welding (2)—welding of thick plate. in Proc. of the 41 st Laser Materials Processing Conference, 21-34 (1997)

9. Y. Kawahito, N. Matsumoto, M. Mizutani, S. Katayama, Characterization of plasma induced during high-power fibre laser welding of stainless steel. Sci. Technol. Weld. Join. 13(8), 744-748 (2008)

10. A. Michalowski, A. Heß, A. Ruß, F. Dausinger, Plume attenuation under high-power Yb:YAG laser material processing. in Proc. 4th
Int. WLT-Conference on Lasers in Manufacturing, LIM, Munich, 357-361 (2007)

11. T. Scholz, K. Dieckmann, A. Ostendorf, Investigation of the formation of nanoparticles during remote welding. Phys. Proc. 41, 90-97 (2013)

12. P.Y. Shcheglov, A.V. Gumenyuk, I.B. Gornushkin, M. Rethmeier, Vapor-plasma plume investigation during high-power fiber laser welding. Laser Phys., 23, 016001 (2013)

13. M. Sohail, S.-W. Han, S.-J. Na, A. Gumenyuk, M. Rethmeier, Role of vapor re-condensation on bead shape in high power laser welding. in Proc. of the 7th International Congress on Laser Advanced Materials Processing (LAMP), paper A010, 1-5 (2015)

14. K. Partes, Analytical model of the catchment efficiency in high speed laser cladding. Surf. Coat. Technol. 204(3), 366-371 (2009)

15. A. Kaplan, A model of deep penetration laser welding based on calculation of the keyhole profile. J. Phys. D Appl. Phys. 27(9), $1805(1994)$

16. H. Zhao, T. DebRoy, Macroporosity free aluminum alloy weldments through numerical simulation of keyhole mode laser welding. J. Appl. Phys. 93(12), 10089-10096 (2003)

17. R. Fabbro, M. Dal, P. Peyre, F. Coste, M. Schneider, V. Gunenthiram, Analysis and possible estimation of keyhole depths evolution, using laser operating parameters and material properties. J. Laser Appl. 30(3), 032410 (2018)

18. F. Fetzer, H. Hu, P. Berger, R. Weber, P. Eberhard, T. Graf, Fundamental investigations on the spiking mechanism by means of laser beam welding of ice. J. Laser Appl. 30(1), 012009 (2018)

19. I. Tomashchuka, M. Mostafab, T. Caudwella, P. Sallamanda, M. Dubanda, 2017. Behavior of laser induced keyhole during dissimilar welding of metals. in Proceedings of Lasers in Manufacturing Conference 2017, Munich, Germany

20. A. Mahrle, M. Borkmann, E. Beyer, C. Leyens, M. Hustedt, C. Hennigs, A. Brodesser, J. Walter, S. Kaierle, Efficient air flow control for remote laser beam welding. in Proc. of the 36th International Congress on Applications of Lasers and Electro-Optics (ICALEO), paper 703 (2017)

21. J.-P. Weberpals, Nutzen und Grenzen guter Fokussierbarkeit beim Laserschweißen. Laser in der Materialbearbeitung, Forschungsberichte der IFSW, T. Graf (Hrsg.), Herbert Utz Verlag (2010)

22. J. Volpp, F. Vollertsen, Keyhole stability during laser weldingpart I: modeling and evaluation. Prod. Eng. Res. Dev. 10(4-5), 443-457 (2016)

23. E.A. Brands, G.B. Brook, Smithells Metals Reference Book (Butterworth-Heinemann Ltd, Oxford, 1992)

24. B.J. Keene, Review of data for the surface tension of pure metals. Int. Mater. Rev. 38(4), 157-192 (1993) 